\title{
MicroRNAs and Diseases: Promising Biomarkers for Diagnosis and Therapeutics
}

\author{
Shailendra Dwivedi ${ }^{1} \cdot$ Purvi Purohit $^{2} \cdot$ Praveen Sharma $^{2}$
}

Published online: 19 July 2019

(C) Association of Clinical Biochemists of India 2019

Human genome draft has shown presence of less than 25,000-28,000 genes in the human genome from such huge genomic contents, so a shift has occurred in the focus of research from mRNAs to noncoding RNAs. MicroRNAs (miRNAs), non-coding short RNA molecules are known hot area of research since past two decades, which regulate gene expression by binding to target mRNAs, resulting in translational repression and gene silencing. Approximately 3000 miRNA molecules have been explored in the mammalian genome, from which over 2000 belong to the human genome. Many major cellular functions such as development, differentiation, growth, and metabolism are known to be regulated by miRNAs. Propinquity to distinct genes in the genome and their locations in introns of coding genes, non-coding genes and exons have been explored to have a major influence on the level of gene expressions in eukaryotic cells. The biogenesis of miRNAs, includes various steps and precise cellular machinery facilitating transcription of miRNAs as small inverted repeats of a double-stranded RNA (dsRNA) stem loop, encompassing 70 bp long and exist in both introns and intergenic clusters in the genome. RNA polymerase II is accountable for the synthesis of the introns and exons of both protein-coding and non-coding transcripts from where miRNAs are generated. Initially, inside the nucleus of the cell, miRNAs are transcribed as primary pri-miRNA transcripts and then are trimmed to form the precursor pre-miRNA stem loop

Praveen Sharma

praveensharma55@gmail.com

1 Department of Biochemistry, All India Institute of Medical Sciences, Gorakhpur 273008, India

2 Department of Biochemistry, All India Institute of Medical Sciences, Jodhpur 342005, India structure before moving into the cytoplasm. Further, inside the nucleus they are processed by the Dicer RNAase III endonuclease and thus mature miRNA of 21-23 nucleotides are produced. The first miRNA lin-4, was discovered in Caenorhabditis elegans in 1993 and then second $C$. elegans miRNA, let-7 but the identification approach was not same. However, the field of miRNA research has since grown with over 17,000 miRNAs discovered to date in 142 species, including humans. The key word "miRNA" currently hits more than 25,000 publications from PubMed, and the first miRNA-targeted drug has now entered a phase II clinical trial (http://www.ClinicalTrials.gov), demonstrating early promise.

As miRNAs are evolutionary conserved from ancestry, and now established as regulatory bio-molecules in various biological pathways, so currently these molecules are being explored in various human diseases [1].

\section{Micro RNAs and Human Diseases}

MicroRNAs have been reported to play a key role in a wide variety of developmental processes including metabolism, cell division, cell proliferation, cell differentiation, apoptosis, development, and even neuronal cell development and differentiation etc. Single point mutations in miRNA or its target or epigenetic silencing of miRNA transcription units is an established mechanism by which the functions of miRNA in cell are affected. Relentless discoveries and fast progress in the previous decades on miRNAs offer the expectation that miRNAs will in the coming future have an immense potential in the diagnosis, prognosis and treatment of various diseases. In the current editorial, a bull's eye view of miRNAs regulatory molecules in different human diseases will be assessed. 
Several studies based on human samples have reported up-regulation or down-regulation of miRNAs in various types of human cancers. Up-regulated miRNAs expression may function as oncogenes and under expressed expression may act like tumor-suppressor genes, and thus regulate various hallmarks of cancer like angiogenesis, apoptosis, cell proliferation, differentiation etc. Results of our project also reported change in dynamics of Breast Cancer Stem cells with oncogenic miRNAs (miR-10 a \& b, miR-21, and miR-17), Tumour suppressor miRNAs (miR-200 a \& b, miR-205 and miR-145) in progression of breast carcinoma. Similarly addition and deletion of any gene fragments harboring miRNAs sequences may cause the development of cancer as in lung cancer, the $5 \mathrm{q} 33$ region harboring miR143 and miR-145 is often deleted, resulting in decreased expression of both miRNAs. On the contrary, augmentation in expression of miR-17-92 cluster gene have been reported in B cell lymphomas and lung cancers and translocation of this cluster gene was also observed in T-cell acute lymphoblastic leukemia.

Transcriptional factor bindings at promoter region of certain miRNAs cluster also affects the development and progression of various cancer, like c-Myc, often upregulated in many malignancies to regulate cell proliferation and apoptosis, activates the transcription of oncogenic miR-17-92 cluster through binding to E-box elements in miR-17-92 promoter. Moreover, c-Myc also represses transcriptional activity of tumor suppressive miRNAs such as mir-15a, miR-26, miR-29, mir-30 and let-7 families and also reciprocally regulates tumor suppressor miR-122 in hepatocellular cancer. Further, epigenetic changes (Hypermethylation/hypo-methylation) of certain miRNAs cluster have been reported, that have shown their role in carcinogenesis like miR-148a, and miR-34b/c cluster when hypermethylated causes silencing in cancer cells by inhibiting their motility, reduced tumor growth and inhibited metastasis formation in vivo. Likewise, miRNAs (miR-9-1, miR-124a and miR-145-5p) which are known to influence tumorigenesis, the declined expressions of these miRNAs is reported due to DNA hypermethylation in breast, lung and colon carcinomas, respectively [2].

Cardiac failure in humans is a major determinant of mortality and morbidity in cardiovascular diseases. The vascular system relies on functioning of endothelial system and angiogenesis, but still molecular mechanisms are not very clear at gene levels. Recently the evidence of microRNAs involvement in regulation of heart function and mammalian functioning of cardiovascular system have revolutionize the research in this field also. Researchers have claimed that three miRNAs (miR-1, miR-133, and miR-208) are over-expressed in the heart, and are chief regulators of heart development and myocyte differentiation. Further, deregulated expression of miR-1 and miR-
133 were reported in human heart failure. Similarly, miR23a, miR-23b, miR-24, miR-195, miR-199a, and miR-214 were found over expressed during cardiac hypertrophy. Moreover, miR-24, miR-125b, miR-195, miR-199a, and miR-214 were also observed upregulated in the tissue of patients with end-stage failing human hearts [3].

Inflammation is a crucial factor of host defense system and a key reaction to infection and injury, which is supposed to be accountable for multiple acute and chronic diseases like Cancer, Metabolic syndrome etc. miR-126 have been shown to inhibit the expression of vascular cell adhesion molecule 1 (VCAM-1), which is vital to intervene leukocyte adherence to endothelial cells. Like, miR-155 has been demonstrated to be induced by cytokines such as TNF- $\alpha$ and IFN- $\beta$ which are well-known mediators of inflammation. Further, miR-146 is known to be induced in macrophages by several microbial components and proinflammatory cytokines. Rheumatoid arthritis is another important inflammatory disorder and two microRNAs (miR-155 and miR-146) have been reported to be significantly over expressed in synovial tissues and synovial fibroblasts.

miRNAs are also explored in neuronal diseases, as they are known to be dynamically regulated in neurogenesis, brain development and also in neuronal plasticity. The exploration at molecular levels of miRNAs related to neurodevelopment disorders [e.g. intellectual disability, autism, Attention Deficit Hyperactivity Disorder (ADHD) and epilepsy] may prove beneficial to the pathogenesis of these disorders. As per recent findings loss of miR-29 cluster has been shown to be associated with increased (beta-amyloid precursor protein-converting enzyme) BACE1/beta-secretase expression in sporadic Alzheimer's Disease patients. Similarly, miR-298 and miR-328 have been identified to directly interact with the 30-UTR of the BACE1 transcript which is involved in beta-amyloid production. More recently, the miR-20a family (i.e. miR-20a, miR-17-5p and miR-106b) has been reported to regulate the expression of Amyloid Precursor Protein (APP) thus provide probable associations for these micro RNAs during Alzheimer's Disease development. Further, in neuropsychiatric disorder of Schizophrenia, role of Brain-Derived Neurotrophic Factor (BDNF) is evident with GABA and glutamatergic neurons and now bioinformatic findings have demonstrated that hsa-mir-1 and hsa-mir-206 have their target of the 30-UTR of BDNF. Thus, this provides a links that miRNA could regulate BDNF protein synthesis by interfering with BDNF mRNA translation during brain development. Finally in bone related diseases, like in osteoporosis, micro RNAs have potential to be robust markers as compared to bone turnover markers (BTM), which are often marred by diurnal variations. More than 50 miRNAs have been identified in human osteoporosis 
studies which target the bone remodeling process including miR-21, miR-23-3p, miR-100, miR-124, miR-125b and $\operatorname{miR}-133[4,5]$.

Thus, not only limited to the above discussed studies, but other studies are also providing evidence that miRNAs play a crucial role in many of developmental processes and diseases. Although no single mechanism can be attributed to unravel their mode of action in various diseases, but consistent research at cellular level supported by robust bioinformatics can largely help decode mysteries of disease pathogenesis $[6,7]$.

In the light of the expansive knowledge gained on role of micro RNAs in disease with every passing day and year, the promise they hold for therapeutics and diagnostics cannot be undermined. Therefore, exploration of miRNAs and its integration with newly developed approaches of miRNA silencing, antisense blocking and miRNA modification may generate new potential in better management of these diseases.

\section{References}

1. Liu B, Li J, Cairns MJ. Identifying miRNAs, targets and functions. Brief Bioinform. 2014;15(1):1-19.

2. Peng Y, Croce CM. The role of MicroRNAs in human cancer. Signal Transduct Target Ther. 2016;1:15004.

3. Samanta S, Balasubramanian S, Rajasingh S, Patel U, Dhanasekaran A, Dawn B, et al. MicroRNA: a new therapeutic strategy for cardiovascular diseases. Trends Cardiovasc Med. 2016;26(5):407-19.

4. Dwivedi S, Purohit P, Misra R, Pareek P, Goel A, Khattri S, et al. Diseases and molecular diagnostics: a step closer to precision medicine. Indian J Clin Biochem. 2017;32(4):374-98.

5. Moran-Moguel MC, Petarra-Del Rio S, Mayorquin-Galvan EE, Zavala-Cerna MG. Rheumatoid Arthritis and miRNAs: a critical review through a functional view. J Immunol Res. 2018;2018:2474529.

6. Sharma P, Dwivedi S. Prospects of molecular biotechnology in diagnostics: step towards precision medicine. Indian $\mathrm{J}$ Clin Biochem. 2017;32(2):121-3.

7. Dwivedi S, Samdariya S, Chikara G, Goel A, Pandey RK, Pareek $\mathrm{P}$, et al. Molecular biotechnology for diagnostics applied molecular biotechnology. In: Khan IA, Barh D, Khan MS, editors. Applied molecular biotechnology. The next generation of genetic engineering. Florida: CRC Press; 2016. p. 303-43.

Publisher's Note Springer Nature remains neutral with regard to jurisdictional claims in published maps and institutional affiliations. 